\title{
Baseline serum cholestanol as predictor of recurrent coronary events in subgroup of Scandinavian simvastatin survival study
}

\author{
Tatu A Miettinen, Helena Gylling, Timo Strandberg, Seppo Sarna, for the Finnish 4S Investigators
}

\begin{abstract}
Objectives: To investigate whether baseline serum cholestanol:cholesterol ratio, which is negatively related to cholesterol synthesis, could predict reduction of coronary events in the Scandinavian simvastatin survival study.

Design: Follow up of patients with coronary heart disease in whom baseline ratios were related to major coronary events.

Setting: Four universities in Finland.

Subjects: A subgroup of 868 patients with coronary heart disease selected from the Scandinavian simvastatin survival study.

Intervention: Treatment with simvastatin or placebo. Main outcome measures: Serum concentrations of low density lipoprotein and high density lipoprotein cholesterol, total triglyceride concentration, and cholesterol:cholestanol ratio. Major coronary events. Results: With increasing baseline quarter of cholestanol distribution the reduction in relative risk increased gradually from $0.623(95 \%$ confidence interval 0.395 to 0.982$)$ to 1.166 (0.791 to 1.72$)$. The risk of recurrence of major coronary events increased 2.2-fold $(\mathrm{P}<0.01)$ by multiple logistic regression analysis between the lowest and highest quarter of cholestanol. The ratio of cholestanol was related inversely to the body mass index and directly to high density lipoprotein cholesterol and triglyceride concentrations but their quarters of distribution were not related to risk reduction.

Conclusions: Measurement of serum cholestanol concentration revealed a subgroup of patients with coronary heart disease in whom coronary events were not reduced by simvastatin treatment. Thus, patients with high baseline synthesis of cholesterol seem to be responders whereas those with low synthesis of cholesterol are non-responders.
\end{abstract}

\section{Introduction}

The Scandinavian simvastatin survival study (4S) showed that simvastatin clearly decreases serum cholesterol concentration and considerably reduces mortality from all causes and coronary events and major coronary events in patients with coronary heart disease. ${ }^{1}$ As the reduction in the relative risk of major coronary events was not associated with the baseline serum lipid concentrations in the survival study, ${ }^{2}$ however, we considered that reduction in relative risk may be related to baseline intestinal absorption or to endogenous synthesis of cholesterol. From among the serum non-cholesterol sterols, the cholesterol precursor sterols-that is, lanosterol and other methyl sterols and demethylated cholestenol, desmosterol, and lathosterol-are directly related to cholesterol synthesis, ${ }^{3-9}$ especially in the liver, ${ }^{10}$ while cholestanol and plant sterols - that is, campesterol and sitosterolare directly related to cholesterol absorption. ${ }^{911}$ Accordingly, we would expect quantification of these sterols to reveal subgroups with high or low absorption or synthesis of cholesterol. To this end we examined whether measurement of baseline serum cholestanol concentration in the Finnish coronary subpopulation of the survival study predicts the extent to which simvastatin could reduce the risk of major coronary events.

\section{Patients and methods}

The whole study population of the survival study and the methods used have been reported previously. ${ }^{12}$ The present study population included the Finnish subgroup of 868 patients with coronary heart disease selected from 1374 candidates for the 4444 participants of the original study and were randomised to placebo $(\mathrm{n}=434)$ or simvastatin $20-40 \mathrm{mg} /$ day $(\mathrm{n}=434)$ for 5 years and 3 months. Distributions of age, sex, and lipid concentrations were similar in the placebo and simvastatin subgroups and were comparable with those in the main study. The secondary end point of the survival study-major coronary events, including coronary deaths, non-fatal myocardial infarctions, and revascularisation procedures-was used for analytical purposes.

Concentrations of total and low and high density lipoprotein cholesterol and triglycerides were analysed at the central laboratory from serum obtained after an overnight fast. ${ }^{12}$ Concentrations of cholesterol and non-cholesterol sterol were measured in two saponified baseline serum samples by gas-liquid chromatography on a $50 \mathrm{~m}$ long SE-30 capillary column (polydimethylsiloxane), with $5 \alpha$-cholestane as an internal standard ${ }^{13}{ }^{14}$ by running the samples from a single patient in a single batch from frozen stored serum samples. The non-cholesterol sterols are transported in serum by lipoproteins, about $70 \%$ by low density lipoprotein, so that the decrease in concentration of low density lipoprotein cholesterol by simvastatin also changes the serum concentration of cholestanol and other non-cholesterol sterols. We have therefore expressed the values of these sterols in terms of $\mathrm{mmol} / \mathrm{mol}$ of cholesterol-that is, as ratios to cholesterol. The values given are means of two baseline determinations.

Statistical analysis-The patients were ranked according to quarters of baseline distribution of body mass index $\left(\mathrm{kg} / \mathrm{m}^{2}\right)$, serum concentrations of total and low density and high density lipoprotein cholesterol, total triglycerides, and cholestanol to cholesterol ratios. We counted major coronary events during the follow up of 5 years 3 months in the quarters of these variables and calculated relative risks $(95 \%$ confidence intervals) between simvastatin and placebo groups. ${ }^{15}$ Analysis of variance was used to compare means of

\section{Department of Medicine, Division of Internal Medicine, University of Helsinki, FI-00290 Helsinki, Finland Tatu A Miettinen, professor \\ Helena Gylling, senior physician Timo Strandberg, assistant professor \\ Department of Public Health, University of Helsinki \\ Seppo Sarna, associate professor Correspondence to: Dr Miettinen tatu.a.miettinen@ helsinki.fi}

BMJ 1998;316:1127-30 
Table 1 Relative risk of major coronary events by simvastatin in patients defined by baseline quarters of total, low density lipoprotein and high density lipoprotein cholesterol and triglyceride concentrations, body mass index, and cholestanol proportion

\begin{tabular}{|c|c|c|c|c|c|c|c|c|}
\hline \multirow[b]{2}{*}{ Variable } & \multicolumn{2}{|r|}{ First quarter } & \multicolumn{2}{|c|}{ Second quarter } & \multicolumn{2}{|r|}{ Third quarter } & \multicolumn{2}{|c|}{ Fourth quarter } \\
\hline & Value & Relative risk (95\% Cl) & Value & Relative risk $(95 \% \mathrm{Cl})$ & Value & Relative risk (95\% Cl) & Value & Relative risk (95\% Cl) \\
\hline Cholesterol (mmol/l) & $<6.0$ & 0.795 (0.538 to 1.17$)$ & $6.0-6.6$ & 0.652 (0.430 to 0.989$)$ & $6.7-7.1$ & $0.953(0.637$ to 1.44$)$ & $>7.1$ & $0.763(0.482$ to 1.21$)$ \\
\hline $\begin{array}{l}\text { Low density lipoprotein } \\
\text { cholesterol (mmol/l) }\end{array}$ & $<4.2$ & $0.683(0.445$ to 1.05$)$ & $4.2-4.6$ & 0.823 (0.528 to 1.28$)$ & $4.7-5.1$ & 0.704 (0.478 to 1.04$)$ & $>5.1$ & 0.926 (0.604 to 1.42$)$ \\
\hline $\begin{array}{l}\text { High density lipoprotein } \\
\text { cholesterol }(\mathrm{mmol} / \mathrm{l})\end{array}$ & $<1.0$ & $0.856(0.609$ to 1.20$)$ & $1.0-1.2$ & $0.639(0.425$ to 0.961$)$ & $1.3-1.4$ & 0.859 (0.550 to 1.34$)$ & $>1.4$ & 0.738 (0.419 to 1.30$)$ \\
\hline Triglycerides (mmol/l) & $<1.1$ & 0.972 (0.674 to 1.40$)$ & $1.1-1.4$ & 0.742 (0.457 to 1.20$)$ & $1.5-1.9$ & 0.681 (0.457 to 1.01$)$ & $>1.9$ & 0.702 (0.447 to 1.10$)$ \\
\hline Body mass index $\left(\mathrm{kg} / \mathrm{m}^{2}\right)$ & $<24.2$ & 0.880 (0.589 to 1.32$)$ & $24.2-26.4$ & $0.737(0.485$ to 1.12$)$ & $26.5-28.4$ & $0.750(0.493$ to 1.14$)$ & $>28.4$ & $0.791(0.523$ to 1.20$)$ \\
\hline $\begin{array}{l}\text { Cholestanol }\left(10^{2} \mathrm{mmol} / \mathrm{mol}\right. \\
{\text { cholesterol })^{\star}}^{*}\end{array}$ & $<107$ & $0.623(0.395$ to 0.982$)$ & $107-126$ & 0.657 (0.426 to 0.998$)$ & $127-148$ & 0.753 (0.502 to 1.130$)$ & $>148$ & $1.166(0.791$ to 1.720$)$ \\
\hline
\end{tabular}

continuous variables in the quarters. Multiple logistic regression analysis was used to test the associations between different prognostic variables and the occurrence of major coronary events. The goodness of fit and consistency with logistic function were evaluated with Hosmer-Lemeshow and C C Brown tests, respectively. The statistical analyses were carried out with BMDP statistical software package. ${ }^{16}$

\section{Results}

In the Finnish subgroup the mean changes in lipid concentrations caused by simvastatin-that is, -28 , $-35,8$, and $-15 \%$ for total, low density lipoprotein, and high density lipoprotein cholesterol and triglycerides, respectively-were similar to those seen in the main study population. ${ }^{1}$

The reduction in the risk of major coronary events in the whole subgroup studied (144 $v 114$ events in placebo $v$ simvastatin group, respectively) was $21 \%$ $(0.790 ; 95 \%$ confidence interval 0.642 to 0.971$)$. When we analysed gradually increasing body mass index and serum lipid concentrations from the first to the fourth quarter only cholestanol:cholesterol ratio exhibited percentages of recurrent events, gradually increasing from $21 \%$ to $35 \%$ (values shown only for cholestanol) with the increasing baseline sterol quarters in the simvastatin but not in placebo group (footnote to table 1). The corresponding relative risk increased gradually from 0.623 (0.395 to 0.982 ) to 1.166 (0.791 to 1.720$)$. Thus, the relative risk of major coronary events was increased by $16.6 \%(-20.9 \%$ to $72.0 \%)$ in the highest quarter of the cholestanol ratio and significantly reduced by $37.7 \%(-60.5 \%$ to $-1.8 \%)$ in the lowest quarter. The concentrations of cholestanol showed inconsistent changes in the relative risk ratios. Age and the concentrations of total and low density lipoprotein cholesterol were similar in all the quarters separated according to the ratios of cholestanol (table 2). The serum concentrations of high density lipoprotein cholesterol increased whereas the serum concentrations of triglycerides and body mass index values decreased. The ratios of cholestanol to cholesterol were negatively related to those of cholesterol precursor sterols (cholestanol, desmosterol, and lathosterol) and strongly positively related to those of plant sterols (campesterol and sitosterol; data not shown).

As in stepwise logistic regression analysis the continuous cholestanol ratio was the only variable of table 1 significantly entered into the model (odds ratio $1.01 ; 1.00$ to 1.02 ), the association between cholestanol and the occurrence of major coronary events was tested by using a fixed logistic model with three variables: treatment group, cholestanol as an ordinal scale variable based on quarters, and treatment group*cholestanol interaction. In this model both the goodness of fit (Hosmer-Lemeshow test, $P$ value 0.983 ) and the consistency of logistic function (C C Brown test, $\mathrm{P}$ value 1.000 ) were good. For the treatment group the odds ratio was 3.29 (1.53 to 7.06) and the Wald's test value $3.05(\mathrm{P}<0.001)$. For cholestanol the odds ratio was 1.31 (1.07 to 1.61). The risk of recurrence of major coronary events increased 2.2-fold between the lowest and highest quarters of the distribution of cholestanol ratios by multiple logistic regression analysis $(\mathrm{P}<0.01$; Wald's test value 2.63). For the interaction term the odds ratio was 0.73 (0.56 to 0.86$)$ and Wald's test $-2.29(\mathrm{P}<0.02)$.

Table 2 Mean (SD) baseline values for different variables defined by quarters of distribution of cholestanol at baseline $(<107,107-126$, $127-148,>148$ )

\begin{tabular}{|c|c|c|c|c|c|c|c|c|}
\hline \multirow[b]{2}{*}{ Variables } & \multicolumn{2}{|c|}{ First quarter } & \multicolumn{2}{|c|}{ Second quarter } & \multicolumn{2}{|c|}{ Third quarter } & \multicolumn{2}{|c|}{ Fourth quarter } \\
\hline & $\begin{array}{c}\text { Simvastatin } \\
(\mathrm{n}=106)\end{array}$ & $\begin{array}{l}\text { Placebo } \\
(n=111)\end{array}$ & $\begin{array}{c}\text { Simvastatin } \\
(n=106)\end{array}$ & $\begin{array}{l}\text { Placebo } \\
(n=111)\end{array}$ & $\begin{array}{c}\text { Simvastatin } \\
(\mathrm{n}=113)\end{array}$ & $\begin{array}{l}\text { Placebo } \\
(\mathrm{n}=104)\end{array}$ & $\begin{array}{c}\text { Simvastatin } \\
(\mathrm{n}=110)\end{array}$ & $\begin{array}{l}\text { Placebo } \\
(n=107)\end{array}$ \\
\hline Age (years) & $58.2(6.9)$ & $58.0(5.8)$ & $57.3(6.9)$ & $57.0(7.4)$ & $58.5(6.3)$ & $58.0(6.4)$ & $58.5(6.4)$ & $58.1(6.2)$ \\
\hline Body mass index $\left(\mathrm{kg} / \mathrm{m}^{2}\right)$ & $28.6(3.7)$ & $28.0(3.9)$ & $27.0(3.5)$ & $26.9(3.6)$ & $26.1(2.6)$ & $26.4(3.1)$ & $25.3(3.1)^{\star}$ & $25.0(2.8)^{\star}$ \\
\hline Cholesterol (mmol/l) & $6.45(0.75)$ & $6.67(0.72)$ & $6.64(0.71)$ & $6.62(0.85)$ & $6.60(0.75)$ & $6.51(0.75)$ & $6.60(0.85)$ & $6.65(0.73)$ \\
\hline LDL cholesterol $(\mathrm{mmol} / \mathrm{l}) \dagger$ & $4.50(0.70)$ & $4.71(0.66)$ & $4.70(0.70)$ & $4.70(0.80)$ & $4.68(0.73)$ & $4.58(0.70)$ & $4.68(0.83)$ & $4.71(0.74)$ \\
\hline HDL cholesterol (mmol/l) & $1.18(0.289$ & $1.15(0.25)$ & $1.19(0.31)$ & $1.19(0.34)$ & $1.25(0.34)$ & $1.21(0.32)$ & $1.32(0.33)^{\star}$ & $1.33(0.33)^{*}$ \\
\hline Triglycerides (mmol/l) & $1.74(0.69)$ & $1.81(0.66)$ & $1.66(0.63)$ & $1.67(0.66)$ & $1.48(0.59)$ & $1.57(0.64)$ & $1.30(0.52)^{*}$ & $1.31(0.60)^{*}$ \\
\hline $\begin{array}{l}\text { Cholestanol }\left(10^{2} \mathrm{mmol} / \mathrm{mol}\right. \\
\text { cholesterol) }\end{array}$ & $91(13)$ & $92(12)$ & $117(6)$ & $116(6)$ & $136(6)$ & $135(6)$ & $171(19)$ & $172(26)$ \\
\hline
\end{tabular}

LDL=low density lipoprotein; HDL=high density lipoprotein.

${ }^{*} P<0.0001$ by analysis of variance.

†Total and LDL cholesterol values differed in first quarter by pairwise comparison but by analysis of variance the two quartile lines were similar.

fLowest cholestanol values in fourth quarters were 148 for both groups. 


\section{Discussion}

Multivariate logistic regression analysis indicated that the baseline lipid concentrations did not contribute to the results shown by the cholestanol ratio. The higher the quarter of the cholestanol ratio the greater was the risk of major coronary events. The risk of recurrence of major coronary events was 2.2-fold between the lowest and highest quarters of cholestanol, a finding not applicable to total, low density lipoprotein, or high density lipoprotein cholesterol. Thus, simvastatin treatment of patients with a low baseline cholestanol ratio predicts a clear reduction in risk of major coronary events, this improvement not being seen in patients with high baseline cholestanol ratio. Similar findings were also observed when the cholestanol ratios were used as continuous or dichotomised variables in statistical analysis. Thus, quantification of baseline cholestanol in patients with coronary heart disease would give important new information to clinicians or practitioners for evaluation of the future success of the statin treatment. The ability to predict the ineffectiveness of the relatively expensive statins in the treatment of coronary patients supports the use of such measurements. Today, automated instrumentation allows comparatively rapid gas liquid chromatographic measurements, each analysis measuring concentrations of cholesterol and several non-cholesterol sterols; the most predictive sterol for evaluation of treatment seems to be cholestanol.

\section{Why resistance to statin treatment?}

Why then are the coronary subjects with high cholestanol ratios resistant to reduction in recurrence of major coronary events? Cholestanol itself is hardly atherogenic, even though simvastatin increases the ratios of cholestanol and plant sterols,${ }^{17}$ phytosterolaemia is strongly atherogenic, ${ }^{18}$ and high plant sterol concentrations may be atherogenic. ${ }^{19}$ The incidence of major coronary events was, however, unrelated to the ratios of cholestanol (or plant sterols) in the placebo group. As already noted, the cholestanol ratios are positively related to absorption and negatively to synthesis of cholesterol, ${ }^{911}$ measured either by the sterol balance technique or cholesterol precursor sterols in serum..$^{3-9} 2021$ Thus, we suggest that patients with high baseline cholestanol ratios do not respond because of high baseline absorption and low synthesis of cholesterol. Preliminary studies showed lower responses induced by simvastatin in serum cholesterol concentrations of subjects with high rather than low baseline cholestanol ratios.

\section{Suggested treatment}

The present findings for the first time relate cholesterol metabolism to statin induced changes in the reduction of the risk of coronary events in secondary prevention. The discovery before treatment of a subgroup of patients who do not respond to statin treatment alone suggests that such patients should be treated by a combination of statin with hypolipidaemic agents increasing cholesterol synthesis-namely, bile acid binding resins or sitostanol ester induced cholesterol malabsorption. ${ }^{22}$ The latter combination decreased low density lipoprotein cholesterol further by about $10 \%{ }^{23}$ and even more when it was combined with statins in
- Recurrence of major coronary events is reduced by statin treatment in about one third of patients with coronary heart disease, to predict those who will not respond has not been possible from baseline lipid concentrations in the Scandinavian simvastatin survival.

- This study showed that increasing quarters of cholestanol:cholesterol ratio, reflecting decreasing synthesis of cholesterol, were related to recurrence of major coronary events during simvastatin treatment in a Finnish subgroup $(n=868)$ of the Scandinavian study.

- The subjects with lowest baseline quarters of cholestanol were associated with significantly reduced relative risk of major coronary events, while the risk in the highest quarter was unchanged and 2.2 times higher than in the lowest one.

- Cholestanol:cholesterol ratios were related inversely to the body mass index and directly to high density lipoprotein cholesterol and triglyceride concentrations, but their quarters were unrelated to risk reduction.

- The findings suggest that patients with coronary disease who have high absorption (high basal cholestanol:cholesterol) and low synthesis of cholesterol do not benefit from statin treatment alone and that they can be identified by measuring serum cholestanol concentration before treatment.

patients with high baseline ratios of cholestanol and plant sterols. $^{24}$

The results of this study were presented at the 11th international symposium on atherosclerosis in Paris, 1997. An abstract is published in Atherosclerosis 1997;134:48(150). The Finnish 4S investigators were TA Miettinen, H Vanhanen, TE Strandberg, K Hölttä, H Luomanmäki, T Pekuri, A Vuorinen (Helsinki University Hospital); A Pasternack, H Oksa, L Siitonen, R Rimpi (Tampere University Hospital); YA Kesäniemi, M Lilja, T Korhonen, A Rantala, M Rantala, M Savolainen, O Ukkola, L Laine, L Virkkala (Oulu University Hospital); K Pyörälä, S Lehto, A Rantala, H Miettinen, A Salokannel, R Räisänen (Kuopio University Hospital).

Contributors: TAM, the guarantor and a member of the steering committee of the survival study, initiated the research, formulated the hypothesis, monitored the analysis of serum sterols, and wrote the paper. HG participated in formulating the hypothesis, collecting and analysing data, and in writing and editing of the paper. TS participated in the preparation of the paper. SS performed the statistical analyses and participated in the preparation of the paper.

Funding: Finnish Academy of Medical Sciences, Finnish Heart Research Foundation, Helsinki University Hospital, Juho Vainio Foundation, and Merck, Sharp, and Dohme. Conflict of interest: None.

1 Scandinavian Simvastatin Survival Group. Randomised trial of cholesterol lowering in 4444 patients with coronary heart disease: the Scandinavian simvastatin survival study (4S). Lancet 1994;344:1383-9.

2 Scandinavian Simvastatin Survival Study Group. Baseline serum cholesterol and treatment effect in the Scandinavian simvastatin survival study (4S). Lancet 1995;345:1274-5.

3 Miettinen TA. Detection of changes in human cholesterol metabolism. Ann Clin Res 1970;2:300-20.

4 Miettinen TA. Serum methyl sterols and their distribution between major lipoprotein fractions in different clinical conditions. Ann Clin Res 1971;3:264-71.

5 Vuoristo M, Miettinen TA. Serum cholesterol precursor sterols in coeliac disease: effects of gluten-free diet and cholestyramine. Gut $1986 ; 27: 1312-9$

6 Gylling H, Miettinen TA. Serum noncholesterol sterols related to cholesterol metabolism in familial hypercholesterolemia. Clin Chim Acta 1988; $178: 41-50$

7 Färkkilä MA, Tilvis RS, Miettinen TA. Raised plasma cholesterol precursors in patients with gut resections. Gut 1988;29:188-95.

8 Kempen HJM, Glatz JFC, Leuven JAG, Voort van der HA, Katan MB. Serum lathosterol concentration is an indicator of whole-body cholesterol synthesis in humans. J Lipid Res 1988;29:1149-55. 
9 Miettinen TA, Tilvis RS, Kesäniemi YA. Serum plant sterols and cholesterol precursors reflect cholesterol absorption and synthesis in volunteers of a randomly selected male population. Am J Epidemiol 1990;131:20-31.

10 Björkhem I, Miettinen TA, Reihner E, Ewerth S, Angelin B, Einarsson K. Correlation between serum levels of some cholesterol precursors and activity of HMG-CoA reductase in human liver. J Lipid Res 1987;28:113743 .

11 Tilvis RS, Miettinen TA. Serum plant sterols and their relation to cholesterol absorption. Am J Clin Nutr 1986;43:92-7.

12 Scandinavian Simvastatin Survival Study Group. Design and baseline results of the Scandinavian simvastatin survival study of patients with stable angina and/or previous myocardial infarction. Am J Cardiol 1993;71:393-400

13 Miettinen TA, Koivisto P. Non-cholesterol sterols and bile acid production in hypercholesterolemic patients with ileal by-pass. In: Paumgartner G, Stiehl A, Gerok W, eds. Bile acids and cholesterol in health and disease. Lancaster, Pennsylvania: MTP, 1983:183-7.

14 Miettinen TA. Cholesterol metabolism during ketoconazole treatment in man. J Lipid Res 1988;29:43-51.

15 Gardner MJ, Altman DG. Confidence interval analysis (CIA): microcomputer program manual and disk. London: BMJ Publishing, 1989.

16 Dixon WJ, ed. BMDP statistical software manual. Vols 1-2. Los Angeles: Berkeley University of California Press, 1992

17 Miettinen TA, Strandberg T, Vanhanen H, Gylling H, for the 4S Group. Non-cholesterol serum sterols in Scandinavian simvastatin survival study. In: Gotto Jr AM, Paoletti R, Smith LC, Catapano AI, Jackson AS, eds. Drugs affecting lipid metabolism. Risk factors and future directions. Dordrecht:
Kluwer Academic Publishers, 1996. (Medical Science Symposium series, No 10:473-6.)

18 Björkhem I, Skrede S. Familial diseases with storage of sterols other than cholesterol: cerebrotendinous xanthomatosis and phytosterolemia. In: Scriver CR, Beaudet AL, Sly WS, Valle D, eds. The metabolic basis of inherited disease. 6th ed. New York: McGraw-Hill, 1989:1283-302.

19 Glueck CJ, Speirs J, Tracy T, Streicher P, Illig E, Vandegrift J. Relationships of serum plant sterols (phytosterols) and cholesterol in 595 hypercholesterolemic subjects, and familial aggregation of phytosterols, cholesterol, and premature coronary heart disease in hyperphytosterolemic probands and their first-degree relatives. Metabolism 1991;40:842-8.

20 Miettinen TA, Kesäniemi YA. Cholesterol absorption: regulation of cholesterol synthesis and elimination and within-population variations of serum cholesterol levels. Am J Clin Nutr 1989;49:629-35.

21 Miettinen TA, Tilvis RS, Kesäniemi YA. Serum cholestanol and plant sterol levels in relation to cholesterol metabolism in middle-aged men. Metabolism 1989;38:136-40.

22 Miettinen TA, Puska P, Gylling H, Vanhanen H, Vartiainen E. Serum cholesterol lowering by sitostanol ester margarine in a mildly hypercholesterolemic random population. N Engl J Med 1995;333:1308-12.

23 Gylling H, Miettinen TA. Effects of inhibiting cholesterol absorption and synthesis on cholesterol and lipoprotein metabolism in hypercholesterolemic non-insulin-dependent diabetic men. J Lipid Res 1996;37:1776-85.

24 Gylling H, Miettinen TA. Sitostanolester added to long term simvastatin treatment of coronary patients with low and high basal cholesterol absorption. Atherosclerosis 1997;134:157.

(Accepted 9 December 1997)

\title{
Risk factors for coronary heart disease and infection with Helicobacter pylori: meta-analysis of 18 studies
}

\author{
John Danesh, Richard Peto
}

Clinical Trial Service Unit and Epidemiological Studies Unit, Nuffield

Department of Clinical Medicine, University of Oxford, Radcliffe Infirmary, Oxford OX2 6HE

John Danesh, Rhodes scholar Richard Peto, professor of medical statistics and epidemiology

Correspondence to: Dr Danesh john.danesh@ balliol.ox.ac.uk

\begin{abstract}
Objective: To find out if chronic infection with Helicobacter pylori is correlated with risk factors for coronary heart disease.

Design: Meta-analysis of 18 epidemiological studies, involving a total of 10000 patients, that measured serum antibody titres to $H$ pylori and risk factors for coronary heart disease. Any study published in any language before 1998 was eligible for inclusion.

Results: Only small absolute differences in body mass index, blood pressure, or haematological risk factors were found between subjects who were seropositive and those who were seronegative. In those who were seropositive body mass index was slightly higher ( 0.37 , SE 0.09) and concentrations of high density lipoprotein cholesterol were slightly lower $(0.032 \mathrm{mmol} / \mathrm{l}, 0.008)$. None of the other differences
\end{abstract} were highly significant.

Conclusion: Previous claims of substantial correlations between $H$ pylori seropositivity and certain vascular risk factors were largely or wholly due to chance or the preferential publication of positive results, or both.

\section{Introduction}

Epidemiological studies have shown that a weakly positive correlation exists between chronic gastric infection with Helicobacter pylori and coronary heart disease. ${ }^{1}$ If this association is causal then infection with $H$ pylori may increase the incidence of coronary heart disease by affecting other vascular risk factors. If there is a non-causal association between $H$ pylori infection and coronary heart disease, then this association must be due to confounding factors. It would be useful to know if infection with $H$ pylori is correlated with body mass index, blood pressure, or haematological factors such as blood lipids, particularly if these variables might also be correlated with coronary heart disease.

When examined individually, the findings of published reports of the possible correlates of $H$ pylori infection seem to have been prone to the effects of chance, or the preferential publication of positive results (publication bias), or both; most studies have had small sample sizes, reported on several different factors, and omitted to perform systematic reviews of the findings of other studies. Systematic reviews of published evidence can increase the amount of data available for analysis; they can also reduce biases that may be introduced through the use of data from small studies that have not been supported by the results of other studies. Such reviews should be less liable to random error and bias than selective emphasis on particular publications would be. We reviewed published studies of the correlations between H pylori seropositivity and variables that might be risk factors for coronary heart disease.

\section{Methods}

Epidemiological and clinical studies in any language published before 1998 that reported on correlations between serum antibody concentrations of $H$ pylori and specific vascular risk factors were identified by searching Medline, relevant reference lists, and gastroenterology and cardiology journals and by discussing studies with the authors of relevant reports. Risk factors examined were systolic blood pressure, diastolic blood pressure, body mass index, plasma viscosity, 